\title{
BMJ Open What influences allied health clinician participation in research in the public hospital setting: a qualitative theory- informed approach
}

Rachel Wenke (D) , ${ }^{1,2,3}$ Christy Noble (D) , ${ }^{4,5}$ Kelly A Weir, ${ }^{1,2,3}$ Sharon Mickan (D) ${ }^{6,7}$

To cite: Wenke R, Noble $\mathrm{C}$, Weir KA, et al. What influences allied health clinician participation in research in the public hospital setting: a qualitative theory-informed approach. BMJ Open 2020;10:e036183. doi:10.1136/ bmjopen-2019-036183

- Prepublication history and additional material for this paper are available online. To view these files, please visit the journal online (http://dx.doi. org/10.1136/bmjopen-2019036183).

Received 04 December 2019 Revised 20 May 2020 Accepted 21 May 2020
Check for updates

(C) Author(s) (or their employer(s)) 2020. Re-use permitted under CC BY-NC. No commercial re-use. See rights and permissions. Published by BMJ.

For numbered affiliations see end of article.

Correspondence to

Dr Rachel Wenke;

rachel.wenke@health.qld.gov.au

\section{ABSTRACT}

Objectives Using theoretical frameworks from

implementation science, we aimed to systematically explore the barriers and enablers to research active allied health professionals (AHP) participating and leading research in the hospital setting.

Design A qualitative interview study informed by behaviour change theory.

Setting Single Australian tertiary hospital and health service.

Participants We recruited a convenience sample of 21 AHPs working within a hospital who were seeking to actively participate in/or lead research within their workplace.

Data collection Semistructured interviews explored perceived barriers and enablers to research participation, informed by the 14 domains of the theoretical domains framework (TDF). Transcribed interviews were deductively coded and mapped to the TDF. A deeper level of inductive coding was used to identify emergent themes that influenced behaviour change, according to the three key constructs of: capability, opportunity and motivation (COM-B).

Results Barriers and enablers to research participation were identified within nine predominant domains of the TDF. Most enablers to engaging in research related to the motivation or opportunity constructs of the COM-B. These enablers included positive beliefs about the consequences of research participation, enabling social influences, peer support and motivation for skill development and to inform practice. Predominant barriers related to environmental context and resources (eg, reduced funding or time), emotional responses of being overwhelmed and perceptions of reduced capability.

Conclusion This study identified key barriers and enablers to behaviour change related to AHPs participating and/or leading research. Motivation and opportunities to participate in research may be enabled by maximising social influence opportunities, reiterating beliefs about positive consequences of research and considering AHP's emotional responses. Implementation science frameworks may provide a more systematic and holistic understanding of factors which influence research participation including enhancing knowledge, motivation and opportunity.
Strengths and limitations of this study

- Our qualitative study is one of the first to use implementation science frameworks to explore barriers and enablers to allied health professionals participating in research.

- We used theory-informed frameworks from implementation science such as the capability, opportunity and motivation model of behaviour system (COM-B) and theoretical domains framework to guide data collection and analyses.

- Participants were from five different allied health professional backgrounds.

- As we involved clinicians from a single public health service, different barriers and enablers may be present within other contexts and sectors.

- There was an over-representation of participants from speech pathology background compared with other professions.

\section{BACKGROUND}

There is a growing body of evidence supporting the importance of health professionals participating in and/or leading research projects, and the benefits this participation provides at individual, organisational and societal levels. ${ }^{1-4}$ Despite the evidence base, further knowledge is needed regarding how to implement this evidence in practice. More specifically, knowledge is needed on how to effectively support health professionals within their clinical context to both 'participate' in (ie, assist with recruitment of participants, data collection or be engaged in research intellectually to sufficiently earn authorship) ${ }^{5}$ and 'lead' research projects (ie, design, source funding, conduct and publish research). ${ }^{5-8}$

Successfully undertaking a research project as a health professional, whether as a participant or leader, is a complex task and requires development of new knowledge and capabilities within their clinical environment. ${ }^{9}$ For example, health professionals may need to 
learn how to integrate new processes into existing clinical pathways to screen for eligible participants, identify and engage with key stakeholders and apply new skills for disseminating findings. Successful implementation of clinician-led research projects may subsequently require health professionals to change their behaviour while developing these capabilities. These behaviour changes can be challenging and are influenced by numerous intrinsic and extrinsic factors including barriers and enablers related to workplace culture, resources and personal motivations. ${ }^{10}{ }^{11}$ It is therefore vital that any barriers to behaviour change, as well as those enablers which productively influence behaviour, are systematically identified and then used to inform interventions to support a health professional's participation or leadership in research projects. Specifically, the use of behaviour change theory within the discipline of implementation science may be helpful to systematically understanding barriers and enablers to research participation. This approach has been effectively used in targeting barriers and encouraging enablers to change in health professional's behaviours in other areas including modifying patient care practices ${ }^{12}$ and use of evidence-based practice behaviours. ${ }^{13}$ As behaviour change theory is a relatively new field, so far there has been very little published literature regarding its application to developing health professionals' capabilities to participate in and/or lead research projects.

\section{Theoretical frameworks and their application}

Implementation science uses frameworks, theories and principles to help translate and implement new knowledge into practice settings, through systematically studying the influences on health professional behaviours to help develop interventions that promote behaviour change. ${ }^{1415}$ Numerous implementation science frameworks exist to help understand behaviour change, with recent discussion papers highlighting the importance of adopting such frameworks and approaches to develop effective change in learner behaviours including in the education of health professionals (eg, medical education). ${ }^{15-17}$ The following study will consider behaviour change in relation to health professional's engagement in research using two key complementary frameworks: the capability, opportunity and motivation model of behaviour (COM-B) system ${ }^{18}$ and the theoretical domains framework (TDF). ${ }^{11}$

The COM-B system was synthesised from 19 frameworks related to designing interventions that promote behaviour change $^{18}$ and describes three essential conditions for behaviour change: capability, opportunity and motivation. Capability refers to 'the individual's psychological and physical capacity to engage in the activity concerned' and includes having the required knowledge and skills ${ }^{18}$ (p 4). Motivation encompasses 'habitual processes, emotional responding, as well as analytical decisionmaking' that 'energize and direct behaviour not just goals and conscious decision-making', while opportunity

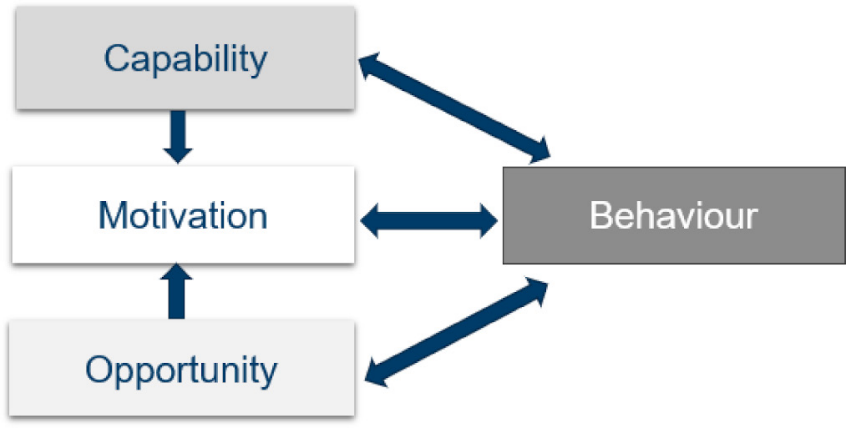

Figure 1 The COM-B system. COM-B, capability, opportunity and motivation model of behaviour.

'is defined as all the factors that lie outside the individual that make the behaviour possible or prompt it ${ }^{18}$ (p 4). The components of the systems and their influences are depicted in figure 1 .

A complementary $\mathrm{TDF}^{9}$ was synthesised from 33 behavioural change theories into 14 domains to analyse the behavioural processes of implementation and inform intervention design. ${ }^{9}$ The framework has been widely used to assist in the understanding of underlying barriers and enablers to implementation problems and subsequently develop interventions to address these issues ${ }^{11}$; however, there has been limited application of these frameworks for facilitating research participation in health professionals.

While research participation of health professionals is growing across all health disciplines, allied health professionals (AHP), comprising approximately a third of the health workforce, ${ }^{19}$ are being increasingly encouraged to participate in research. ${ }^{20-22}$ Allied health encompasses several health professions including physiotherapy, social work, occupational therapy, dietetics, speech languagepathology, podiatry, psychology and pharmacy among others, with often only basic research training being provided as part of their graduate qualifications. Previous research has investigated general barriers and enablers to AHPs' engagement in research in the workplace. Many of these studies surveyed the general workforce and did not seek to specifically understand the perspectives of healthcare clinicians who were conducting a research project, ${ }^{23-25}$ or were not underpinned by theory-informed frameworks such as the COM-B or TDF. ${ }^{10}{ }^{23-25}$ Subsequently, these studies may have overlooked underlying factors impacting the development of new behaviours.

More recently, Stewart et $a l^{26}$ surveyed 136 pharmacists in remote and rural health board areas in Scotland applying the TDF to explore pharmacists' views and experiences of conducting, disseminating and translating research. The authors concluded that while most pharmacists were interested in research, the 'knowledge' and 'environmental context and resources' domains of the TDF were perceived as barriers to research conduct and dissemination. ${ }^{26}$ The authors suggested behaviour change techniques addressing knowledge should be targeted at an individual level (eg, regarding available funding 
opportunities, training and organisational research priorities), while strategies should be undertaken at an organisational level to address issues related to the 'environmental context and resources, ${ }^{26}$

While Stewart et $a l^{26}$ used a theoretically informed approach to deepen understanding of influences that enable or hinder research participation, further investigation of such influences for other types of health professionals and healthcare contexts will help inform intervention strategies to support behaviour change associated with health professional's research participation. ${ }^{18}$ Considering this, the following research aimed to describe, analyse and synthesise the barriers and enablers to behaviour change in AHPs who are participating in and leading research within an Australian tertiary hospital and health service.

\section{METHODS}

\section{Study design}

We used a qualitative interview-based study informed by behaviour change theory ${ }^{11} 18$ and a content and thematic analysis approach to analysing the in-depth interview. This study constitutes the first phase of a larger study with the protocol previously published..$^{27}$ The larger study aimed to understand how allied health research fellows use specific strategies to facilitate allied health clinician's engagement in research. The current phase involved interviewing clinicians who were participating and/ or leading research to help inform the development of tailored strategies to facilitate their research engagement.

\section{Setting}

The research took place within a large tertiary regional health service in Queensland, Australia. While the primary aim of the health service is to provide publicly funded healthcare to patients within a non-metropolitan region, the health service also recognises the value of research within the organisation. As such, the health service encourages health professionals across disciplines to be actively engaged in research including using research to inform practice, participating in research projects others are leading, as well as leading and initiating their own research.

\section{Participants}

We invited allied health staff who were interested in actively participating and/or leading clinical research projects within the health service to participate in the study. This comprised a convenience sample of allied health staff who responded to an initial Expression of Interest distributed by email from their managers to receive support from an allied health research fellow to undertake the research activity that they were either participating in or leading. All participants had their line manager's approval and were employees of the health service during their participation.

\section{Data collection}

In 2016-2017, semistructured interviews exploring participants' perceived barriers and enablers to participating in, and/or leading research were conducted at a time and location convenient to the interviewees by one of three allied health research fellows (RW, CN or KW). The interview was generally in a meeting room or office close to the participant's work area. The interviewer, in most cases, had interacted or had some form of professional relationship with the interviewees prior to the interview. All of the allied health research fellows were female and held a PhD with an interest in research capacity building. Two fellows had a speech pathology clinical background, and one had a pharmacy background, and all had experience working as a clinician-researcher during their careers, with RW currently working part-time as a clinician in the health service.

Participants were provided with an information sheet about the purpose of the interview and had the opportunity to review and reflect on the broad questions prior to the interview. The semistructured interview guide (see online supplementary file 1) and suggested prompts were designed to explore the participant's barriers and enablers to engaging in research, across all 14 domains of the TDF as described in online supplementary file $2 .{ }^{11}{ }^{28}$ Interviews were conducted either in a group of two or more participants (where health professionals were working on the same research project) or single interviews. Interviewers took field notes during the interview for reflexivity, however they were not used in final analyses.

\section{Data analyses}

Digital audio recordings of interviews were transcribed by a professional transcription service. Two levels of data analyses were undertaken. First, barriers and enablers from transcribed interviews were mapped to the TDF using deductive coding in $\mathrm{NVivo}^{29}$ as per processes detailed in Atkins et al. ${ }^{9}$ This involved three authors (KW, $\mathrm{CN}$ and RW) deductively coding informational meaning units to an agreed domain of the TDF and identifying whether it was reported as a 'barrier' or 'enabler', and further developing subcategories within these categories using an inductive approach. Additional comments that were not considered a barrier or enabler were also coded using inductive coding within the TDF categories and discussed as a team; however, as these isolated comments were not related to the research question, they were not included in the final presentation of the findings. Due to the similarity between intentions and goals domains of the TDF in the data collected, these two domains were merged into one. For the second level of analyses, RW further synthesised the subcategories identified within the TDF domains into overarching themes in order to summarise the key findings of the interviews. As reported in tables 1 and 2, this included approximately 80 subcategories across both barriers and enablers being grouped into common themes. The themes were then mapped onto the three components of the COM-B system ${ }^{18}$ : capability, opportunity and motivation, to give a broader understanding of the influences on behaviour change and to inform future development of learning interventions. ${ }^{9}$ This process was guided by the pre-set mapping 
Table 1 Enablers to research participation and leadership from TDF

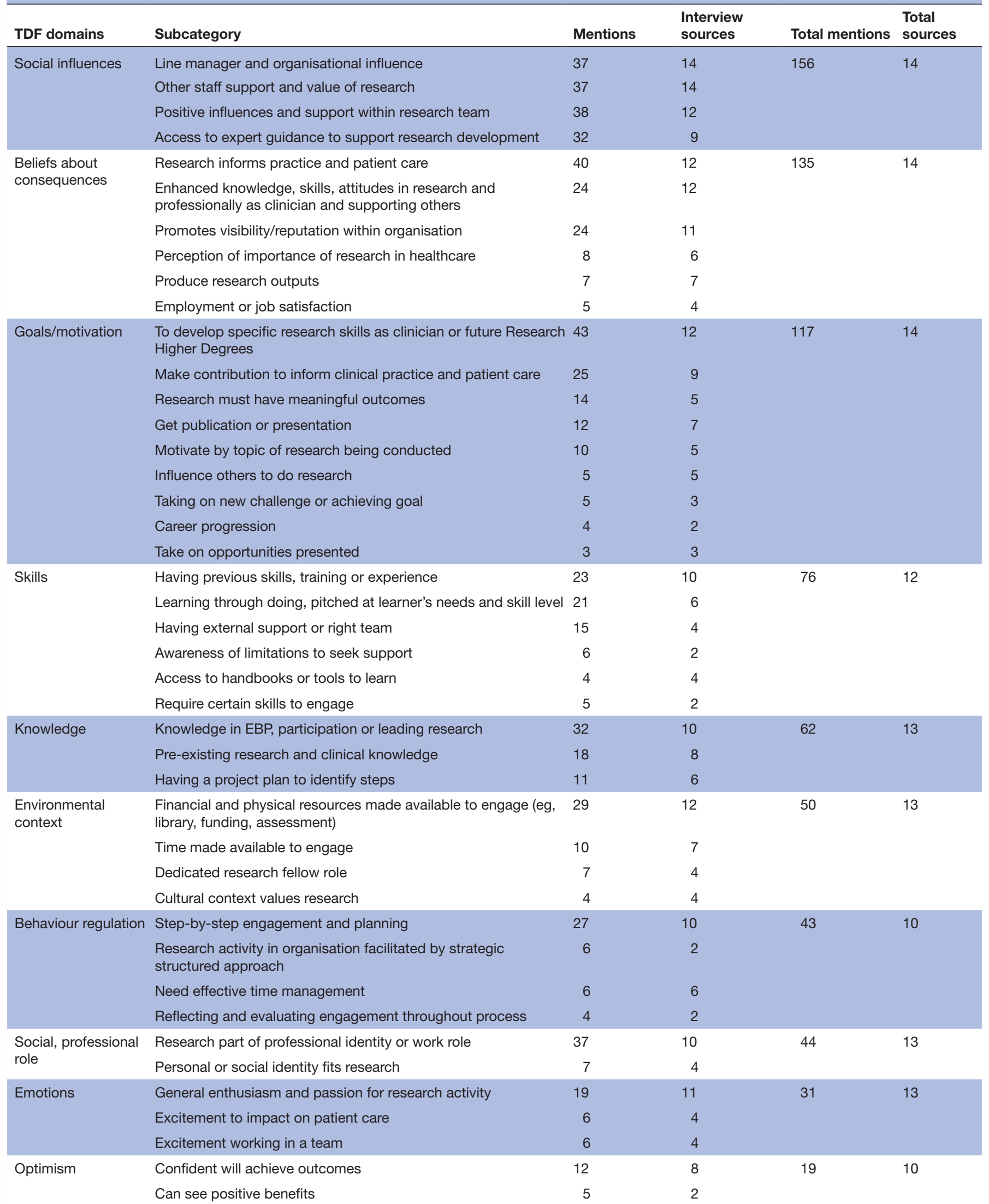

Continued 
Table 1 Continued

\begin{tabular}{|c|c|c|c|c|c|}
\hline TDF domains & Subcategory & Mentions & $\begin{array}{l}\text { Interview } \\
\text { sources }\end{array}$ & Total mentions & $\begin{array}{l}\text { Total } \\
\text { sources }\end{array}$ \\
\hline \multirow{3}{*}{$\begin{array}{l}\text { Beliefs about } \\
\text { capabilities }\end{array}$} & Believes one has capability to engage in research & 6 & 3 & \multirow[t]{3}{*}{14} & \multirow[t]{3}{*}{8} \\
\hline & Feeling confident to undertake research with support & 4 & 2 & & \\
\hline & Believes previous skills will be useful & 4 & 3 & & \\
\hline $\begin{array}{l}\text { Memory, decision- } \\
\text { making }\end{array}$ & Getting in right head space, flexibility with problem solving & 7 & 4 & 10 & 4 \\
\hline Reinforcement & $\begin{array}{l}\text { Regular support, increasing knowledge, interest on topic, seeing } \\
\text { outcomes }\end{array}$ & 6 & 1 & 6 & 1 \\
\hline
\end{tabular}

EBP, Evidence based practice; RHD, Research higher degree; TDF, theoretical domains framework.

\begin{tabular}{|c|c|c|c|c|c|}
\hline Domain & Subcategory & Mentions & Sources & $\begin{array}{l}\text { Total } \\
\text { mentions }\end{array}$ & $\begin{array}{l}\text { Total } \\
\text { sources }\end{array}$ \\
\hline \multirow[t]{4}{*}{ Environmental context } & Time or funding pressures & 37 & 11 & 104 & 13 \\
\hline & Coordinating activity with staff or service changes & 33 & 8 & & \\
\hline & Competing clinical or research priorities & 23 & 10 & & \\
\hline & Reduced access to research resources & 7 & 8 & & \\
\hline \multirow[t]{4}{*}{ Emotions } & $\begin{array}{l}\text { Feeling overwhelmed of nervousness or intimidation in undertaking } \\
\text { research }\end{array}$ & 29 & 11 & 40 & 13 \\
\hline & Fear of getting it wrong & 7 & 6 & & \\
\hline & Coping with uncertainty & 4 & 5 & & \\
\hline & Frustration with longer time frames for research & 3 & 2 & & \\
\hline \multirow{3}{*}{$\begin{array}{l}\text { Beliefs about } \\
\text { capabilities }\end{array}$} & Perceived lack of self-efficacy in general aspects of research & 28 & 9 & 42 & 11 \\
\hline & Lack of confidence in statistics and data analysis & 11 & 5 & & \\
\hline & Lack of confidence in presenting research & 2 & 2 & & \\
\hline \multirow[t]{2}{*}{ Behaviour regulation } & $\begin{array}{l}\text { Balancing tension of juggling research with other work and distractions } \\
\text { (ie, email) }\end{array}$ & 12 & 7 & 24 & 7 \\
\hline & Having to prioritise clinical work over research work & 12 & 3 & & \\
\hline \multirow{4}{*}{$\begin{array}{l}\text { Beliefs about } \\
\text { consequences }\end{array}$} & May miss other job opportunities & 7 & 3 & 20 & 7 \\
\hline & Research will be restrictive or less valuable & 7 & 2 & & \\
\hline & Difficulties with recruitment or other operational constraints & 3 & 2 & & \\
\hline & May miss seeing patients & 2 & 2 & & \\
\hline \multirow[t]{2}{*}{ Social influences } & Perception of negative opinion or support in operational team & 9 & 4 & 12 & 5 \\
\hline & Managers not engaging in research or giving lip-service & 3 & 3 & & \\
\hline \multirow[t]{2}{*}{ Skills } & Lack of skills learnt at university & 6 & 4 & 12 & 6 \\
\hline & Lack of skills limit ability & 2 & 2 & & \\
\hline \multirow[t]{3}{*}{ Goals/motivation } & $\begin{array}{l}\text { When the research idea is not initiated by clinician but someone else or } \\
\text { in unrelated area }\end{array}$ & 4 & 2 & 8 & 2 \\
\hline & Lack of clarity or vision for the research & 2 & 1 & & \\
\hline & Reduced motivation & 2 & 1 & & \\
\hline \multirow[t]{2}{*}{ Knowledge } & Lack of knowledge of processes & 6 & 4 & 8 & 5 \\
\hline & Lack of time to access knowledge & 2 & 1 & & \\
\hline \multirow{2}{*}{$\begin{array}{l}\text { Social, professional } \\
\text { role and identity }\end{array}$} & Challenge of straddling between two roles & 4 & 2 & 9 & 4 \\
\hline & Personal social circumstances may impact on engagement & 3 & 2 & & \\
\hline \multirow{2}{*}{$\begin{array}{l}\text { Memory, attention, } \\
\text { decision-making }\end{array}$} & Prioritisation of other tasks & 5 & 2 & 7 & 2 \\
\hline & Staff needing reminders to collect data & 2 & 1 & & \\
\hline Optimism & Pessimism and feeling guilty about lack of progress & 3 & 3 & 3 & 3 \\
\hline
\end{tabular}

TDF, theoretical domains framework. 
relationship between the COM-B and TDF (see online supplementary file 3) outlined by Cane et al. ${ }^{11}$

\section{Findings}

Twenty-three allied health staff responded to the Expression of Interest (sent by managers to approximately 800 allied health staff) and were invited to interview. Twenty-one staff participated in the interviews as two were unavailable. Participants identified as speech pathologists $(n=10)$, occupational therapists $(n=3)$, social workers $(n=2)$, dieticians $(n=2)$, physiotherapists $(n=2)$ or therapy assistants $(n=2)$. They were mostly senior clinicians $(n=9)$, as well as team leaders $(n=6)$, entry-level clinicians $(n=3)$, therapy assistants $(\mathrm{n}=2)$ and a director of an allied health profession $(n=1)$. Over half of participants $(n=12)$ were leading research projects (ie, conceptualising research protocol, leading ethics application, leading writing for publication) and were considered a principal investigator, while the remaining $(n=9)$ were participating in research projects through assisting with data collection or participant recruitment on a project that was being led by other collaborators. While some of the participants had been involved in other research projects, all were considered 'novice' researchers and had no formal postgraduate research qualifications. Interviews ranged from 20 to $60 \mathrm{~min}$ (median $44 \mathrm{~min}$ ) and included 10 one-on-one interviews and four group interviews.

Participants described barriers and enablers to research participation across all domains of the TDF and reported almost twice as many enablers. Subcategories within the 14 domains are listed in order of frequency of mention in tables 1 and 2, with the number of interview sources also provided. Further descriptions of the names of the categories of the 14 TDF domains are found in online supplementary file 2. Although participants referred to all 14 domains of the TDF, most comments related to nine of these domains, which will now be described as they relate to the three components of behaviour: capability, opportunity and motivation of the COM-B system. ${ }^{11}$ TDF categories described within each of these constructs are based on pre-set relationships between the COM-B and the TDF (as per online supplementary file 3). Figure 2 further synthesises the key themes of the TDF domains within the three constructs of the COM-B which was performed as part of the secondary analyses.

\section{Motivation}

Barriers and enablers which related to motivation for participating and leading research included beliefs about consequences, goals, emotions and beliefs about capabilities.

\section{Beliefs about consequences and goals Enablers}

All participants believed that participating in research would lead to 'better patient outcomes' (P15) and noted that setting and achieving research goals was likely to be a key enabler to engaging in research. One clinician commented, 'I know that it's going to have potential impacts on how the service

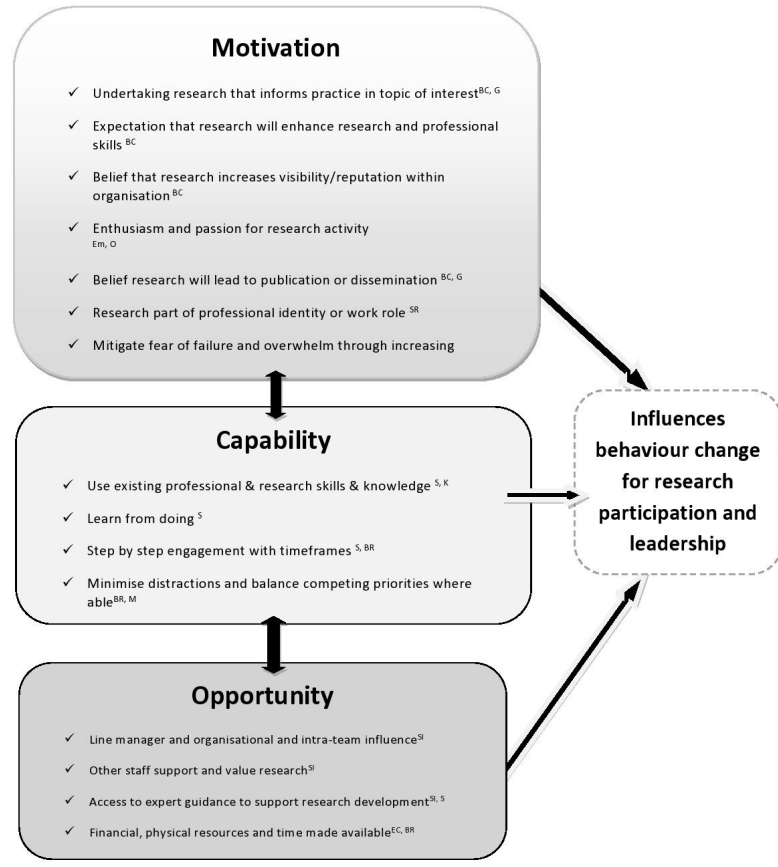

Figure 2 Key factors which influence behaviour change for clinician research participation and leadership from thematic analysis. BC, beliefs about consequences; BR, behaviour regulation; EC, environmental context and resources; Em, emotions; G, goals; K, knowledge; M, memory, attention and decision-making; O, optimism; S, skills; SI, social influences; SR, social, professional role and identity.

is going to be delivered and how to make clinical practice bettermore efficient, more coordinated' (P04). Clinicians also noted their personal goals for developing specific research skills as being an important enabler, 'it's a wonderful project to actually build our own research capacity and our links with universities and I also hope that it will personally build my research skills so I can then better support others to engage in other research as well' (P12). Another positive belief about consequences was that clinicians also reported that participating in research would enhance their profession's reputation, 'I think [our profession] has quite a good reputation at the moment for engaging in research, and I think it will just continue to improve that, and improve our profile... I think that's a great thing, not only for us but for our patients...' (P15).

\section{Emotions}

Across 13 out of 14 interviews, clinicians described their emotional responses to research as both an enabler and barrier to participating or leading research.

\section{Barrier}

Clinicians described feeling overwhelmed or intimidated at the thought of undertaking research, 'I think it's just how overwhelming it is...It does seem really mysterious, and it does seem like you've got to be really, really, really clever to do $i t^{\prime}$ (P15). Across six interviews, clinicians described a fear of failure, 'I don't want to do all this work and... be seen as a failure by them or [as] someone who's not clever enough or intelligent enough to be able to do research' (P07). 


\section{Enabler}

Despite this fear, participants across 11 out of 14 interviews expressed a general enthusiasm or passion for the research they were undertaking or about to undertake. For example, one clinician described being 'really excited at the prospect of it' (P03).

\section{Beliefs about capabilities \\ Barrier}

A barrier reported by clinicians across almost $80 \%$ of interviews was a lack of belief or confidence in their capability to undertake aspects of research, with clinicians from five interviews reporting this particularly in relation to statistics and data analyses, 'Statistical analysis I am useless with' (P07).

\section{Enabler}

While clinicians from three interviews reported having some confidence in their ability to undertake research as an enabler, 'I know in myself that I have the ability to do this... I've done some research before' (P10), others reported their self-confidence or belief in their own capability improved with mentoring, 'definitely each time we pretty much meet and discuss things further, I feel slightly more confident' (P04).

\section{Opportunity}

Barriers and enablers which related to opportunities to research participation/leadership included the social influences and the environmental context and resources.

\section{Social influences}

\section{Enablers}

All participants identified social influences as an enabler to participating in research. Specifically, they described the positive influence of line manager and organisational support, 'I'm really lucky that because my manager is very supportive of our team undertaking research, I don't really think having time offline or any of that sort of thing would be as much of an issue' (P15), as well as being surrounded by other staff who support or value research, 'I think when people do research all the time, you're around those people, it starts to feel like more like something you should be doing and it feels more natural...' (P11).

\section{Barrier}

Less commonly, clinicians reported that colleagues had a negative opinion of their participation, 'I'm getting a lot of judgement too, from other people in my department... who haven't necessarily done research themselves who feel that I should have progressed more than what I have' (P10).

\section{Environmental context and resources \\ Barriers}

Most clinicians described pressures associated with limited time or funding to undertake research as a barrier. Coordinating components of research activity within the context of available staffing and service-based changes were also described as difficult, 'I'm in a position where it's very challenging, because I'm not easily backfilled...we haven't really had anyone that can just step into my role and do $i t$ ' (P07), as well as finding the time to complete research amidst a busy case load, 'we've both had very busy caseloads which makes it difficult to...fit much in on non-research days. I think we're both well aware that if we need more time it won't come during work time, it will come at home' (P04).

\section{Enablers}

Amidst discussion of these barriers, enabling aspects of the environmental context were also described across all interviews. This included having the 'funding and having the dedicated time to actually undertake the project' (P16), and a dedicated research fellow role 'supporting and mentoring through the process' (P01).

\section{Capability}

Barriers and enablers related to capability needed to participate/and or lead research included the TDF domains of skills, knowledge and behavioural regulation.

\section{Skills and knowledge \\ Enablers}

Clinicians across 10 of the interviews reported that previous research skills, training or experience enabled their participation in research and included both existing research skills, 'I've done an honours project before, so I've had a bit of background in research' (P05), and professional skills such as 'time management' and 'building networks' (P15). Many clinicians also described 'learning through doing' (P13) as an enabler. Most clinicians also reported having an awareness or knowledge of what is involved in participating in and/or leading research.

\section{Barrier}

Some more experienced clinicians reported that a lack of research-related skills learnt at a university was a barrier to their current participation in research, 'I notice a difference in generations. I went through university when you barely had a computer...' (P07). Clinicians also reported lacking knowledge in more practical areas around research processes within the health service. 'I currently have to go to... someone else and constantly be saying, what do I do with this, how do I do that. I really would like to know those answers and be able to answer them for other people as well (P16).

\section{Behavioural regulation}

\section{Enablers}

Most clinicians (from 10 out of 14 interviews) reported that measures that regulate behaviour including setting clear time frames and sequential tasks enabled their participation in research, 'I think a trick is to break it down to smaller goals and have timeframes to set and hold myself accountable to and to regularly spend time on my research and make that a priority no matter what' (P10).

\section{Barriers}

Clinicians from half of the interviews described the tension of other distractions as a barrier, 'That's probably the biggest challenge for me is that focus, when you've always got 
people calling you and emailing you because you're trying to do two jobs' (P11).

\section{DISCUSSION}

This qualitative study has used implementation science frameworks to explore barriers and enablers to AHPs participating in research to help understand what influences the necessary behaviour changes needed to undertake research projects in the clinical environment. We identified factors across all behavioural domains of the TDF that influenced the motivation, opportunity and/ or capability of AHPs undertaking research, with most comments pertaining to nine of these domains.

The present study was the first to use an implementation science approach to gain a better understanding of the processes of behaviour change required for AHPs to participate in or lead research. This approach may support the newer models of education for health professionals such as medical education models ${ }^{15}$ which focus on 'not just knowledge acquisition (which is necessary but not sufficient) but behaviour change and improvement $^{15}$ ( $p$ 9). Indeed, our study revealed that the most commonly reported factors influencing research participation related to motivation of the AHP as opposed to their knowledge acquisition or capability. While previous research in AHPs has commented on the importance of enhancing motivating factors to support research productivity, ${ }^{30}$ there remains a lack of attention in the literature regarding strategies to specifically address motivation. A recent realist review of research capacitybuilding interventions classified most interventions being either training, environmental restructuring or mentoring, thereby likely targeting the development of capability and opportunity rather than motivation. ${ }^{7}$ To enhance motivation including reinforcing beliefs about consequences and goals, the authors of the 'behaviour change wheel' suggest using interventions which involve persuasion, incentivisation, modelling and enablement. ${ }^{18}$ Another realist review evaluating what constitutes a successful research environment also has suggested that to enhance their motivation, health professionals should also consider reflecting on reasons why they identify as researchers, as they 'develop a researcher identity such that their research becomes more internally motivated rather than just externally driven, ${ }^{, 31}$ ( $p$ 11).

While enablers to motivation were apparent in the interviews, a key barrier to motivation towards research participation was emotions, including fear of failure and feeling overwhelmed. The pervasiveness of this theme may be related to the fact that our present research only included participants who were already actively involved or about to be in research, in contrast to other studies which sampled from a wider population that may have included health professionals who were not considering active involvement in research. ${ }^{32} 33$ As such, the reality of undertaking the unknown and its associated fears may have been more of an immediate reality in our sample of participants. A similar emotion was experienced by residents and alumni on residency programmes who reported having anxiety about the uncertainty surrounding their research activity expectations. ${ }^{34}$ Participants in the present study however reported that stepby-step guidance from a more experienced researcher such as a research facilitator alleviated these negative emotions. This is in alignment with the control value theory of achievement emotions, which suggests that the amount of emotional and cognitive support provided to a person to enhance the perceived control and value in a task can facilitate one's positive emotions towards achieving an outcome. ${ }^{35}$ While the role and mechanisms for research facilitators have been explored previously including providing individual mentoring, supporting infrastructure development and developing research agendas for health professionals working in health settings, ${ }^{36-38}$ little has been reported regarding their role in supporting positive emotions associated with health professional research participation.

The social influence of expert guidance from a research facilitator, and the manager and organisational support were also seen as predominant enablers to participation in research, enabling the social opportunity to undertake research. The positive impact of manager and organisational support ${ }^{739}$ and the presence of expert guidance on research participation ${ }^{2} 32373840$ have been previously reported but not in the context of providing social opportunities to enhance behaviour change in health professionals. The enabling impact of social influences from other peers, team members and researchers may support the programme theory introduced by Cooke $e t a l^{7}$ which describes modelling of positive behaviour by others involved in research as a key mechanism to enhancing research engagement.

While we employed implementation science theories in the present study, our findings support the results of a realist review which used workplace learning theory ${ }^{40}$ to explore strategies that support research activities for medical doctors and may suggest a complementary relationship between workplace learning theory and implementation science. Indeed, many of the factors identified as contexts and mechanisms of the realist review overlapped with our findings. ${ }^{40}$ For example, Noble et al reported the context as having appropriate 'opportunities' to engage in practice-informed research and having protected time and funding. The value of clear practice curriculum with effective sequencing or a step-by-step approach to learning and practice pedagogies (including 'learning through doing') which support a health professional's 'capability' was also addressed in the review ${ }^{40}$ in similarity with our study. Noble's review also highlighted the importance of a person's readiness to learn, being linked to 'motivation'; however, as few papers in the review addressed this area, the authors suggested this is an area requiring further research. 


\section{Limitations}

A limitation of the present study is that it involved AHPs from a single public health service and as such, different barriers and enablers may be present within other contexts and sectors (ie, private vs public). While recruitment was open to all allied health professions, participants were from five professions only, and thus did not represent all allied health professions. Moreover, a substantial proportion $(\sim 48 \%)$ of participants were from the speech pathology profession. Therefore, caution may be required in translating our findings to other professional groups. While all participants were considered 'novice' researchers with no tertiary research qualifications, information regarding previous research experience was not collected and may have influenced individual responses to interviews.

\section{Educational implications and recommendations for future research}

Important implications can be drawn from this research for health professionals who are considering undertaking or currently undertaking research, and their mentors and managers. Healthcare organisations seeking to increase research participation, as well as academics and educators seeking further inquiry in this area should also consider these implications. First, we demonstrated that use of theory-informed frameworks from implementation science such as the COM-B system and TDF is valuable in helping to understand and address barriers to research participation in health professionals. Use of these frameworks allows a more systematic and holistic understanding of factors which influence research participation including knowledge, and motivation and opportunity. Likewise, use of these theories assisted in identifying factors and mechanisms underlying enablers to research. Using this comprehensive process of analysis, including frameworks such as the COM-B and TDF is recommended when planning and researching tailored learning programmes for health professionals. Once a barrier and its corresponding COM-B construct are identified, intervention functions such as enablement modelling or environmental restructuring ${ }^{18}$ can be identified with a subsequent practical strategy either from the literature or experience to implement that function, as outlined in table 3. For example, if negative emotions associated with research are an underlying barrier to research participation then strategies of enablement addressing this barrier need to be incorporated into the health professional's research development plan. Specifically, findings from this research will be used to help develop tailored strategies that research fellows will use to support clinicians undertaking research as part of a larger research project. ${ }^{27}$

From a health organisational perspective, the study further revealed that managers should consider the importance of social influences in building AHPs' motivation to engage in research and attempt to maximise these influences to encourage research participation. Investing in dedicated research positions to provide expert guidance, hosting clinicians who are undertaking research within proximity to one another and creating opportunities for AHPs undertaking research to network and offer peer support to one another may facilitate behaviour changes.

Second, research mentors should consider strategies which foster a high degree of control and value for health professionals which may support positive emotions and subsequent motivation needed to participate in research. ${ }^{35}$ Artino et $a l^{35}$ provide examples of strategies including clearly structuring activities, using a step-bystep goal-centred approach, allowing time for the health

\begin{tabular}{|c|c|c|c|}
\hline $\begin{array}{l}\text { Influencing barrier or } \\
\text { enabler }\end{array}$ & $\begin{array}{l}\text { Construct of } \\
\text { COM-B }\end{array}$ & $\begin{array}{l}\text { Intervention } \\
\text { function(s) }{ }^{18}\end{array}$ & Strategies to support behaviour changes needed for research participation \\
\hline $\begin{array}{l}\text { Reduce negative } \\
\text { emotions }\end{array}$ & Motivation & Enablement & $\begin{array}{l}\text { Consider strategies which foster a high degree of control and value. }{ }^{35} \\
\text { Clearly structuring activities and expectations using a step-by-step goal- } \\
\text { centred approach. }{ }^{35} \\
\text { Allow time for health professional's self-paced learning. }{ }^{35} \\
\text { Acknowledging expressions of negative emotions. }{ }^{35} \\
\text { Valuing and recognising effort, risk taking and creativity. }\end{array}$ \\
\hline $\begin{array}{l}\text { Reinforce positive } \\
\text { beliefs about } \\
\text { consequences }\end{array}$ & Motivation & $\begin{array}{l}\text { Modelling } \\
\text { Enablement }\end{array}$ & $\begin{array}{l}\text { Mentors and managers should reinforce positive beliefs about the } \\
\text { consequences of research including informing practice and increasing team } \\
\text { profile. }\end{array}$ \\
\hline
\end{tabular}

AHP, allied health professional; COM-B, capability, opportunity and motivation model of behaviour. 
professional's self-paced learning, acknowledging expressions of negative emotions, and valuing and recognising effort, risk taking and creativity. Third, as much research initiated in clinical practice is never disseminated, mentors and managers should reinforce positive beliefs that AHPs may hold about the benefits of research in clinical practice, including informing practice and increasing of team profile, to support ongoing motivation to progress research through to publication. Advocating for financial and physical resources to continue opportunities to support ongoing participation and completion of research activities is also recommended. Future research should explore the credibility and transferability of these behaviour theory-informed recommendations to determine their usefulness in supporting health professionals in participating in research.

\section{CONCLUSION}

Implementation science frameworks which seek to understand behaviour changes in health professionals such as the COM-B and TDF may be useful to understand factors influencing AHPs' participation in research to develop interventions which support research participation. Greater focus needs to be given to supporting AHP's motivation to participate in research, including reiterating clinician's beliefs about the positive consequences of research and considering the clinician's emotional response to research when providing mentorship. Interventions which enhance these factors, as well as maximising social influences should be prioritised in future research capacity-building investigations.

\section{Author affiliations}

${ }^{1}$ Allied Health Services, Gold Coast Hospital and Health Service, Southport, Queensland, Australia

${ }^{2}$ School of Allied Health Sciences, Griffith University, Gold Coast, Queensland, Australia

${ }^{3}$ Menzies Health Institute Queensland, Griffith University, Gold Coast, Queensland, Australia

${ }^{4}$ Office of Medical Education, Faculty of Medicine, University of Queensland,

Brisbane, Queensland, Australia

${ }^{5}$ School of Medicine, Griffith University, Gold Coast, Queensland, Australia

${ }^{6}$ Faculty of Health Sciences and Medicine, Bond University, Gold Coast, Queensland, Australia

${ }^{7}$ Nuffield Department of Primary Care Health Sciences, University of Oxford, Oxford, UK

Twitter Rachel Wenke @racheljwenke, Christy Noble @chnoble and Sharon Mickan @Smickan

Acknowledgements We thank all clinicians who offered their time to participate in this research.

Contributors SM conceptualised the design of this research with support from $\mathrm{CN}, \mathrm{KW}$ and RW. KW, RW and $\mathrm{CN}$ collected the interview data and undertook the first phase of data analyses. RW led the secondary data analyses and the write-up of the manuscript with all the authors providing critical input and revision of the manuscript drafts.

Funding The authors have not declared a specific grant for this research from any funding agency in the public, commercial or not-for-profit sectors.

Patient and public involvement Patients and/or the public were not involved in the design, or conduct, or reporting, or dissemination plans of this research.
Patient consent for publication Not required.

Ethics approval Ethical clearance was gained from the local Health Human Research Ethics Committee before the study was commenced (approval number HREC/16/QGC/96). All participants provided informed consent.

Provenance and peer review Not commissioned; externally peer reviewed.

Data availability statement Data are available upon reasonable request.

Open access This is an open access article distributed in accordance with the Creative Commons Attribution Non Commercial (CC BY-NC 4.0) license, which permits others to distribute, remix, adapt, build upon this work non-commercially, and license their derivative works on different terms, provided the original work is properly cited, appropriate credit is given, any changes made indicated, and the use is non-commercial. See: http://creativecommons.org/licenses/by-nc/4.0/.

\section{ORCID iDs}

Rachel Wenke http://orcid.org/0000-0003-2100-4898

Christy Noble http://orcid.org/0000-0001-8763-234X

Sharon Mickan http://orcid.org/0000-0002-5690-1997

\section{REFERENCES}

1 Lizarondo L, Grimmer-Somers K, Kumar S. A systematic review of the individual determinants of research evidence use in allied health. $J$ Multidiscip Healthc 2011;4:261-72.

2 Williams C, Miyazaki K, Borkowski D, et al. Research capacity and culture of the Victorian public health allied health workforce is influenced by key research support staff and location. Aust Health Rev 2015;39:303-11.

3 Boaz A, Hanney S, Jones T, et al. Does the engagement of clinicians and organisations in research improve healthcare performance: a three-stage review. BMJ Open 2015;5:e009415.

4 Hanney S, Boaz A, Jones T, et al. Engagement in research: an innovative three-stage review of the benefits for health-care performance. Health Ser Deliv Res 2013;1:1-152.

5 Del Mar C, Askew D. Building family/general practice research capacity. Ann Fam Med 2004;2 Suppl 2:S35-40.

6 Matus J, Walker A, Mickan S. Research capacity building frameworks for allied health professionals - a systematic review. BMC Health Serv Res 2018;18:716.

7 Cooke J, Gardois P, Booth A. Uncovering the mechanisms of research capacity development in health and social care: a realist synthesis. Health Res Policy Syst 2018;16:93.

8 Bates I, Boyd A, Aslanyan G, et al. Tackling the tensions in evaluating capacity strengthening for health research in low- and middleincome countries. Health Policy Plan 2015;30:334-44.

9 Atkins L, Francis J, Islam R, et al. A guide to using the theoretical domains framework of behaviour change to investigate implementation problems. Implement Sci 2017;12:77.

10 Harvey D, Plummer D, Nielsen I, et al. Becoming a clinician researcher in allied health. Aust Health Rev 2016;40:562-9.

11 Cane J, O'Connor D, Michie S. Validation of the theoretical domains framework for use in behaviour change and implementation research. Implement Sci 2012;7:37.

12 Shrubsole K, Worrall L, Power E, et al. The acute aphasia implementation study (AAIMS): a pilot cluster randomized controlled trial. Int J Lang Commun Disord 2018;53:1021-56.

13 Campbell L, Novak I, Mclntyre S, et al. A KT intervention including the evidence alert system to improve clinician's evidence-based practice behavior - a cluster randomized controlled trial. Implement Sci 2013;8:1-28.

14 Eccles MP, Mittman BS. Welcome to implementation science. Implement Sci 2006;1:1.

15 Price DW, Wagner DP, Krane NK, et al. What are the implications of implementation science for medical education? Med Educ Online 2015;20:27003.

16 Thomas DC, Berry A, Djuricich AM, et al. What is implementation science and what forces are driving a change in medical education? Am J Med Qual 2017;32:438-44.

17 Carney PA, Crites GE, Miller KH, et al. Building and executing a research agenda toward conducting implementation science in medical education. Med Educ Online 2016;21:32405.

18 Michie S, van Stralen MM, West R. The behaviour change wheel: a new method for characterising and designing behaviour change interventions. Implement Sci 2011;6:42.

19 Nancarrow SA, Young G, O'Callaghan K, et al. Shape of allied health: an environmental scan of 27 allied health professions in Victoria. Aust Health Rev 2017;41:327. 
20 Pickstone C, Nancarrow S, Cooke J, et al. Building research capacity in the allied health professions. Evid Pract 2008;4:53-68.

21 Borkowski D, McKinstry C, Cotchett M, et al. Research culture in allied health: a systematic review. Aust J Prim Health 2016;22:294-303.

22 Hulcombe J, Sturgess J, Souvlis T, et al. An approach to building research capacity for health practitioners in a public health environment: an organisational perspective. Aust Health Rev 2014;38:252-8.

23 Plummer D, Pighills A, Pain T. Practitioner research capacity: a survey of social workers in Northern Queensland AU - Harvey, Desley. Australian Social Work 2013;66:540-54.

24 Howard AJ, Ferguson M, Wilkinson P, et al. Involvement in research activities and factors influencing research capacity among dietitians. $J$ Hum Nutr Diet 2013;26 Suppl 1:180-7.

25 Holden L, Pager S, Golenko X, et al. Validation of the research capacity and culture (RCC) tool: measuring RCC at individual, team and organisation levels. Aust J Prim Health 2012;18:62-7.

26 Stewart D, Cunningham S, Strath A, et al. A theoretically informed survey of the views and experiences of practicing pharmacists on research conduct, dissemination and translation. Res Social Adm Pharm 2019:15:1298-308.

27 Mickan S, Wenke R, Weir K, et al. Strategies for research engagement of clinicians in allied health (stretch): a mixed methods research protocol. BMJ Open 2017;7:e014876.

28 American Psychological Association. APA dictionary of psychology. Washington: American Psychological Association, 2007.

29 NVivo. Qualitative data analysis [program] 10 version, 2012. Available: https://www.qsrinternational.com/nvivo-qualitative-dataanalysis-software/home

30 Wenke RJ, Mickan S, Bisset L. A cross sectional observational study of research activity of allied health teams: is there a link with selfreported success, motivators and barriers to undertaking research? BMC Health Serv Res 2017;17:114.
31 Ajjawi R, Crampton PES, Rees CE. What really matters for successful research environments? A realist synthesis. Med Educ 2018;52:936-50.

32 Pager S, Holden L, Golenko X. Motivators, enablers, and barriers to building allied health research capacity. $J$ Multidiscip Healthc 2012:5:53-9.

33 Matus J, Wenke R, Hughes I, et al. Evaluation of the research capacity and culture of allied health professionals in a large regional public health service. J Multidiscip Healthc 2019;12:83-96.

34 Ledford CJW, Seehusen DA, Villagran MM, et al. Resident scholarship expectations and experiences: sources of uncertainty as barriers to success. J Grad Med Educ 2013;5:564-9.

35 Artino AR, Holmboe ES, Durning SJ. Control-value theory: using achievement emotions to improve understanding of motivation, learning, and performance in medical education: AMEE guide No. 64 Med Teach 2012;34:e148-60.

36 Wenke R, Mickan S. The role and impact of research positions within health care settings in allied health: a systematic review. BMC Health Serv Res 2016;16:355.

37 Wenke RJ, Ward EC, Hickman I, et al. Allied health research positions: a qualitative evaluation of their impact. Health Res Policy Syst 2017:15:6

38 Ward EC, Hargrave C, Brown E, et al. Achieving success in clinically based research: the importance of mentoring. J Med Radiat Sci 2017;64:315-20.

39 Golenko X, Pager S, Holden L. A thematic analysis of the role of the organisation in building allied health research capacity: a senior managers' perspective. BMC Health Serv Res 2012;12:276.

40 Noble C, Billett SR, Phang DTY, et al. Supporting resident research learning in the workplace: a rapid realist review. Acad Med 2018;93:1732-40. 